\title{
Correction to: Case report of cryptogenic multifocal ulcerous stenosing enteritis (CMUSE): a rare disease may contribute to endoscopy-capsule retention in the small intestine
}

En-Wei Tao, Tian-Hui Zou, Yong-Feng Wang, Jie-Ting Tang, Ying-Xuan Chen and Qin-Yan Gao*

Correction to: BMC Gastroenterol

https://doi.org/10.1186/s12876-019-0962-8

Following publication of the original article [1], the author reported the wrong version of Table 1 has been published. The word of 'Capsule' was mistakenly written as 'Capusle'. The correct version of the table can be found below:

The original article has been corrected as well.

Received: 11 April 2019 Accepted: 11 April 2019

Published online: 29 April 2019

\section{Reference}

1. Tao, et al. Case report of cryptogenic multifocal ulcerous stenosing enteritis

(CMUSE): a rare disease may contribute to endoscopy-capsule retention in the small intestine. BMC Gastroenterol. 2019;19:49. https://doi.org/10.1186/ s12876-019-0962-8.

\footnotetext{
* Correspondence: gaoqinyan@hotmail.com

Division of Gastroenterology and Hepatology, Shanghai Institute of Digestive

Disease, State Key Laboratory for Oncogenes and Related Genes, Key

Laboratory of Gastroenterology \& Hepatology, Ministry of Health, Ren-Ji

Hospital, Shanghai Jiao-Tong University School of Medicine, 145 Middle

Shandong Road, Shanghai 200001, China
}

(C) The Author(s). 2019 Open Access This article is distributed under the terms of the Creative Commons Attribution 4.0 International License (http://creativecommons.org/licenses/by/4.0/), which permits unrestricted use, distribution, and reproduction in any medium, provided you give appropriate credit to the original author(s) and the source, provide a link to the Creative Commons license, and indicate if changes were made. The Creative Commons Public Domain Dedication waiver (http://creativecommons.org/publicdomain/zero/1.0/) applies to the data made available in this article, unless otherwise stated. 
Table 1 Clinical characteristics of the patients diagnosed with CMUSE

\begin{tabular}{|c|c|c|c|c|c|c|c|c|c|c|c|}
\hline \multirow{2}{*}{$\begin{array}{l}\text { No./Sex/ } \\
\text { Age(yr) }\end{array}$} & \multicolumn{4}{|l|}{ Clinical symptoms } & \multicolumn{3}{|c|}{ Laboratory examinations } & \multicolumn{2}{|c|}{ Radiologic examinations } & \multirow[t]{2}{*}{ Location of lesion } & \multirow[t]{2}{*}{ Origin } \\
\hline & Abdominal pain & Anemia & Diarrhea & Fever & $\overline{\mathrm{Hb}}(\mathrm{g} / \mathrm{L})$ & Alb (g/L) & FOBT & $\overline{\mathrm{SBS}}$ & Abdominal CT & & \\
\hline \multicolumn{12}{|c|}{ Capsule retention } \\
\hline $1 / F / 21$ & Y & Y & N & N & 34 & 16 & + & / & Thickening & Ileum & Case in China \\
\hline $2 / F / 30$ & $\mathrm{~N}$ & Y & N & Y & 58 & 20.1 & - & / & Thickening & Ileum & Case in China \\
\hline $3 / M / 44$ & Y & Y & N & N & 45 & 28 & + & Strictures & Dilatation & Jejunum & {$[28]$} \\
\hline $4 / F / 10$ & N & Y & $\mathrm{N}$ & N & 80 & 18.3 & - & / & / & Ileum & [24] \\
\hline $5 / M / 41$ & Y & Y & $\mathrm{N}$ & N & 55 & 30 & + & / & Thickening & Ileum & {$[15]$} \\
\hline $6 / F / 36$ & Y & Y & $\mathrm{N}$ & N & 6.6 & Mild & + & / & Normal & Ileum & {$[15]$} \\
\hline 7/M/52 & Y & Y & Y & N & 60 & / & / & Strictures & Normal & Ileum & {$[25]$} \\
\hline \multicolumn{12}{|c|}{ No capsule retention } \\
\hline $8 / M / 25$ & Y & $\mathrm{N}$ & $\mathrm{N}$ & N & Normal & 24 & - & Strictures & / & Duodenum + Jejunum & [29] \\
\hline 9/M/29 & Y & N & $\mathrm{N}$ & N & Normal & / & - & / & Normal & Ileum & {$[26]$} \\
\hline $10 / F / 29$ & Y & Y & $\mathrm{N}$ & N & 66 & 20.8 & + & / & Thickening & Ileum & {$[23]$} \\
\hline $11 / F / 35$ & Y & Y & Y & N & 96 & 35.8 & / & / & / & Jejunum + lleum & [3] \\
\hline $12 / F / 60$ & $\mathrm{~N}$ & Y & Y & Y & 94 & 15.2 & / & / & / & Jejunum & [3] \\
\hline
\end{tabular}

I: Not done or clearly described; FOBT Fecal occult blood test, SBS Small bowel series, CT Computed tomography 